\title{
Effect of an ac electric field on weak electron localization in Bi films
}

\author{
B. 1. Belevtsev, J. Hasse*, A. S. Anopchenko, and E. Yu. Beliayev \\ B. Verkin Institute for Low Temperature Physics \& Engineering of the National Academy of Sciences of Ukraine, \\ 47 Lenin Ave., 310164 Kharkov, Ukraine
}

* Physikalisches Institut, Universität Karlsruhe, Engesserstr. 7,76128 Karlsruhe, Germany

E-mail:hasse@fphvax.physik.uni-karlsruhe.de

Submitted July 20, 1995

\begin{abstract}
We have studied the effects of weak localization and electron-electron interaction in the conductivity of Bi films at temperatures between 1.1 and $4.2 \mathrm{~K}$ in the presence of a high-frequency electric field $(\approx 9.4 \mathrm{GHz})$. The experiments were carried out under conditions of no appreciable overheating of electrons due to a microwave radiation. The data obtained permit us to examine the influence of temperature on quantum corrections $\Delta \sigma(\Omega)$ at high-frequency electric field more comprehensively and reliably than in earlier works. The experimental dependences $\Delta \sigma(\Omega, T)$ are found to be in good agreement with the known theoretical models.
\end{abstract}

\section{Introduction}

The behavior of the conductivity of disordered metal systems may depart considerably from the classical Drude model at sufficiently low temperatures. The deviation is connected with the wave nature of electrons and depends on the quantum effects of weak localization (WL) and electron-electron interaction (EEI) $[1,2]$. The WL effects are due to the fact that at low temperatures the conduction electrons can be scattered repeatedly by impurities (or other crystal lattice defects), which, however, does not disturb their phase coherence. If the impurity concentration is high enough, the interference of the scattered waves generated by each collision with the impurities increases the probability of back-scattering. At sufficiently low temperatures this results in a negative temperature coefficient for the resistance. The corresponding quantum correction to the conductivity of two-dimensional systems is then logarithmically dependent on temperature $(\Delta \sigma \propto \ln T)$. It is important that the value of the quantum correction is directly controlled by the electron phase relaxation time $\tau_{\varphi}$. The character and frequency of the phase-breaking processes are dictated by inelastic electron-electron and electron-phonon collisions, as well as by the spinflip scattering.

The phase of the electron is considerably influenced by the external magnetic field. The quantum correction related to the WL effect is therefore quite sensitive to the static magnetic field. The times of inelastic $\left(\tau_{i}\right)$, spin-orbit $\left(\tau_{s o}\right)$ and spin-spin $\left(\tau_{s}\right)$ electron relaxations can be derived from experimental studies of the dependence $\Delta \sigma(H)$. This maintains a continuous interest in the WL and EEI effects.
It is known $[1,3]$ that the WL and EEI effects are greatly influenced by the ac (unlike dc) electrical field. In the WL case this occurs once the ac field $E_{a c}(t)$ has its component along the electron trajectory and acts upon the electron, thus changing its wave phase. According to Ref. 3, the mean phase shift during the time $t$ is

$$
\langle\Delta \varphi\rangle \simeq e^{2}\left(d E_{a c}(t) / d t\right)^{2} D t^{5}=\alpha(\Omega t)^{5},
$$

where $\alpha=4 e^{2} D E_{a c}^{2} / \hbar^{2} \Omega^{3} ; D$ is the electron diffusion coefficient; $\Omega$ and $E_{a c}$ are the angular frequency and amplitude of the electric field. The field influence on the phase of the electron is effective only if the field $E_{a c}(t)$ varies considerably during the time $\tau_{\varphi}$. Fields of the frequencies $\Omega \geq \tau_{\varphi}^{-1}$ are therefore required to observe dependence of WL effects on the ac electric field.

To describe the influence of the ac electric (and static magnetic) field on the WL and EEI effects, theoretical models were developed $[1,3]$ which permit direct comparison between theory and experiment. The WL and EEI effects in the magnetic field were the subject of numerous publications but experimental works studying the influence of the ac electric field can easily be counted on one's fingers [4-9]. This is mainly because such studies are complex, effort-consuming and run into difficulties in finding the true amplitude $E_{a c}$ in the samples, which is required for quantitative comparison.

Earlier studies of the influence of a high-frequency electric field upon the quantum corrections to conductivity were made on films $[4,5,7-9]$ and inversion layers $[6]$ and concentrated on the resistance varia- 
tions (using a dc or low-frequency field) in the external high-frequency field. The influence of the ac electric field $E_{a c}$ on the WL effects was not always detected clearly [4], since the field $E_{a c}(t)$ can simultaneously cause considerable Joule heating of the samples. On the other hand, the overheating effects may be used to calibrate the amplitude $E_{a c}$ in the samples, using the equation

$$
\Delta \sigma\left(E_{a c}\right)=\Delta \sigma\left(E_{d c}\right)
$$

where the lettwand and the right-hand sides show conductivity variations in the ac and dc electric fields, respectively. The calibration is performed when the ac field influence on the WL effects is negligible (in high magnetic fields $[6]$ or for high-resistance samples with short phase-breaking time $\tau_{\text {, }}(7-9)$, taking it into account that $L \ll \delta$ ( $L$ and $\delta$ are the film thickness and skin depth, respectively). It was believed $[7-9]$ that the calibration results taken on high-resistance filmes could be applied to low-resistance samples to separate the contributions of the $E_{\text {ac }}(t)$ field to quantum corrections and the Joule heating. This calibration method appeared to be effective for $S b$ films with high sheet resistance $R_{a}$ $(>100 \Omega) \quad 17,81$ (good agreement with theory to within a unity factor). It was, however, completely ineffective for AuPd and Au films $[7,9]$, where it gave an unexpected result: in low-resistance $\left(R_{\mathrm{iI}} \leq 100 \Omega\right)$

films the temperature rise provoked by the Joule heating in a high-frequency field is much smaller than that in a low-frequency field of the same amplitude. The authors of Refs. 7,9 failed to explain the discrepancy. We attribute this result to the erroneous approach 191 underlying the above calibration method, which assumes that the field $E_{a c}(t)$ remains the same in different samples, irrespective of their conductivity (or $R_{12}$ ) $[10 \rrbracket$. It is known [11] that the reflectivity of the electromagnetic wave from the metal is dependent on its conductivity. Electrodynamic calculation shows $[10]$ that at $L<<\delta$ the amplitude of the ac electric field is indeed uniform inside the sample. Its value is, however essentially dependent on $R_{\mathrm{n}}$ and may be considerably lower than the pre-assigned amplitude $E_{\mathrm{ext}}$ of the incident electromagnetic wave. This may account for the apparent inefficiency of the Joule heating in a high-frequency field that was observed for low- $R_{\mathrm{U}}$ films [9]. Numerical calculation supports this conclusion [10].

Experimental studies of the influence of a high-frequency electric field upon the WL and EEI effects are actually at the initial stage and many questions remain to be answered $[4-9]$. For example, no adequate attention has been paid to the temperature depundence of the influence exerted by a high-frequen- cy electric field. Meantime, this kind of investigation could give as reliable information about various mechanisms of inelastic and spin relaxation of electrons as that derived from the studies of the WL and EEI effects in the magnetic field. Further studies and accumulation of experimental data are required to attain this methodical level. In this context the present work was aimed at studying the influence of the highfrequency electric $(9.4 \mathrm{GHz})$ field upon the WL effects in bismuth films. The choice of the experimental objects was dictated by the fact that the WL and EEI effects are well studied in Bi films (Refs. 12,13 and the References therein), which aids the identification of the ac electric field action on these quantum effects.

\section{Experimental technique}

TwO Bi films ( $\mathrm{Bi}-1$ and $\mathrm{Bi}-2$ ) having sheet resistances of $\simeq 357$ and $\simeq 401 \Omega$, respectively, were investigated. The purity of source material was $99.999 \%$. The Bi-1 film (thickness $L=15 \mathrm{~nm}$ ) was deposited in vacuum via thermal evaporation (the pressure of residual gases $P=2 \cdot 10^{-4} \mathrm{~Pa}$ ) with deposition rate $v_{c}=0.07 \mathrm{~nm} / \mathrm{s}$. The parameters of the $\mathrm{Bi}-2$ film were $L=27.6 \mathrm{~nm}, P=1.3 \cdot 10^{-3} \mathrm{~Pa}, v_{\mathrm{c}}=0.3 \mathrm{~nm} / \mathrm{s}$. The substrates were polished single-crystal sapphire plates $(0.5 \times 7 \times 24 \mathrm{~mm})$. Transverse gold films (over $100 \mathrm{~nm}$ thick) were deposited onto the plate ends to serve as electrical contacts in the measurement of the film resistance. The contact widths were $1.2 \mathrm{~mm}$, so the measured length of the films was $21.5 \mathrm{~mm}$, the film widths were $0.065 \mathrm{~mm}(\mathrm{Bi}-1)$ and $0.95 \mathrm{~mm}(\mathrm{Bi}-2)$.

The influence of the microwave field on the film conductivily was investigated in a conventional glass cryostet with ${ }^{4} \mathrm{He}(1.1-4.2 \mathrm{~K})$ using a superconducting lead resonator with the resonance frequency $9.3548 \mathrm{GHz}$. The sapphire plate with the film was inserted into the microwave cavity through two rectangular slots $(\approx 7 \times 1 \mathrm{~mm})$ so that the gold contacts were outside. The plate was fixed from outside with a nonconducting adhesive. Four wires were attached to the gold contacts with the conducting adhesive Eccobond $56 \mathrm{C}$ (Grace Electronic Materials Company) for measurements of the film resistance. A low-frequency ac bridge (AC Resistance bridge $L R-700$, Linear Res. Inc., San Diego, CA, USA) was used. The relative experimental error was not more than $10^{-5}$. In the external high-frequency field the resonator with the sample was immersed in liquid helium.

After the microwave measurements, the same samples were examined in a vacuum cryostat with a superconducting solenoid where the sapphire plates with the deposited films were in good thermal contact with a copper ${ }^{3} \mathrm{He}$ vessel. The measurement of the quantum corrections to the conductivity as a function of the perpendicular magnetic field $H(0-40 \mathrm{kOe})$ and temperature $(0.5-50 \mathrm{~K})$ permitted an independent 
estimation of the phase relaxation times of the electrons. The measuring dc currents $(1-10 \mu \mathrm{A})$ were chosen, which excluded possible influence of the Joule neating. In this case the relative error of the resistance measured also did not exceed $10^{-5}$.

\section{Results and discussion}

The low temperature behavior of the conductivity in the films investigated is controlled by the WL and EEI effects. The temperature dependence of the resistance $R(T)$ has a minimum at $T=5 \mathrm{~K}$. Below this temperature the resistance obeys the logarithmic law $\Delta R \propto \ln T$ (Fig. 1). According to $\mathrm{WL}$ and EEI theories $[1,2]$, the temperature dependence of resistance in a zero magnetic field can be described as

$$
\frac{\Delta R}{R}=-a_{T} \frac{e^{2} R_{\mathrm{n}}}{2 \pi^{2} \hbar} \ln T+\text { const },
$$

where the dimensionless coefficient $a_{T}$ in different limiting cases is

$$
a_{T}=\left\{\begin{array}{l}
1+p \text { at } \tau_{s o} \gg \tau_{i}(T) ; \tau_{s} \gg \tau_{s o}, \tau_{i}(T), \\
1-\frac{p}{2} \text { at } \tau_{s o} \ll \tau_{i}(T) ; \tau_{s} \gg \tau_{s o}, \tau_{i}(T), \\
1 \quad \text { at } \tau_{s} \ll \tau_{i}(T) .
\end{array}\right.
$$

Here $\tau_{i}$ is the phase relaxation time due to inelastic scattering processes; $\tau_{i}^{-1} \propto T^{p}$ ( $p$ is the index dependent on the mechanism of inelastic scattering); $\tau_{s}$ and $\tau_{\text {so }}$ are the electron relaxation times due to the spin-spin and spin-orbit interactions (SSI and SOI). In Eqs. (4) the WL and EEI contribution are repre-

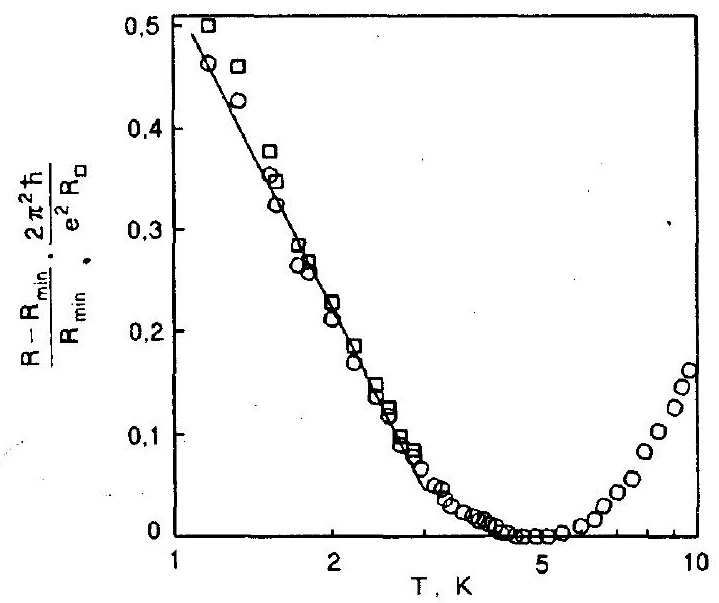

Fig. I. The plots of resistance versus temperature (in the reduced coordinates corresponding to $\mathrm{Eq}$. (3)) for sample $\mathrm{Bi}-1\left(R_{\min }\right.$ is the film resistance at the minimum point of the temperature dependence $R(T)$ ). Experimental points $\square$ and $\bigcirc$ correspond to the presence and absence of applied high-frequency electric field. sented by the $p$-dependent term and the term equal to unity, respectively.

As was shown earlier (Refs. 12,13 and References therein), Eq. (4b) is obeyed for Bi films, which corresponds to the case of strong SOI and weak electron scattering by magnetic impurity; hence $a_{T}=1-p / 2$ is obeyed. For the films studied $a_{T} \simeq 0.45$, which is close to 0.5 , and thus gives $p \simeq 1$. We obtained the same value of $p$ directly from the measurements of
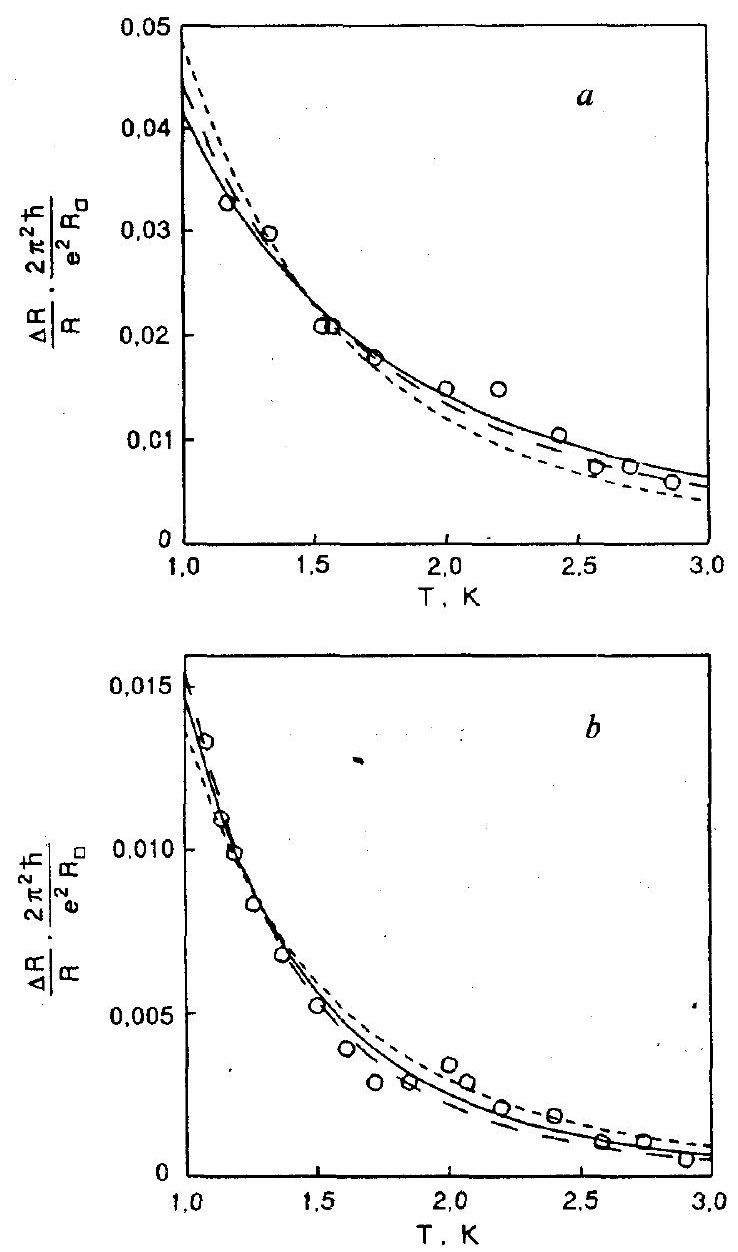

Fig. 2. The temperature dependences of fractional changes in resistance $\triangle R / R$, induced by high-frequency electric field, for $\mathrm{Bi}-1$ (a) and $\mathrm{Bi}-2$ (b). The curves drawn through the experimental points were calculated by $\mathrm{Eq}$. (7) for the correction $\Delta \sigma_{W L}(\Omega)$. In doing so, the values of $D$ and $\tau_{\varphi}(T)$ were prescribed and the values of coefficient $\alpha$ and consequently the amplitude $E_{a c}$ were fitting parameters. The values of $\tau_{\varphi}(T)$ were calculated by the equation $\tau_{\varphi}^{-1}(T)=A T$, where for $A$ we used our experimental values $A_{\text {exp }}$ (long-dashed line), the theoretical values $A_{t h}$ obtained from Eq. (12) (short-dashed line) and the calculated values $A_{\text {th }}$ obtained from Eq. (12) (line) and the calculated values $A_{\text {calc }}$ obtained with varying the values of $A$ and $\alpha$ simultaneously on accomplishing the best agreement between the $\mathrm{Eq}$. (7) and the experimental data (solid line). The values of $D$ were determined by the film resistivity (see below). The values $A_{\exp }, A_{\text {th }}$ and $A_{\text {calc }}$ as well as those of $\alpha$ and $E_{a c}$ for different variants of calculation are listed in Table 1. 
quantum corrections to conductivity in the magnetic field (discussed below).

When a high-frequency electric field was applied, the film resistance increased (Figs. 1,2). As theory postulates [3], the influence of the ac electric field on the WL quantum correction to conductivity can be described as

$$
\Delta \sigma_{W L}(\Omega)=\Delta \sigma(\Omega)-\Delta \sigma(0)=\frac{3}{2} f_{1}\left(\tau_{\varphi}^{*}\right)-\frac{1}{2} f_{1}\left(\tau_{\varphi}\right),
$$

where $\tau_{\varphi}^{*-1}=\tau_{s o}^{-1}+\tau_{\varphi}^{-1}$, and $\tau_{\varphi}^{-1}=\tau_{i}^{-1}(T)+2 \tau_{s}^{-1}$ (for $\operatorname{Bi}_{\varphi} \tau_{\varphi}^{-1} \simeq \tau_{i}^{-1}(T)$ ), and

$$
\begin{gathered}
f_{1}\left(\tau_{\varphi}\right) \equiv-\frac{e^{2}}{2 \pi^{2} \hbar} \times \\
\times \int_{0}^{\infty} \frac{d x}{x} \exp \left(\frac{-4 x}{\Omega \tau_{\varphi}}\right)\left\{\exp \left[-\alpha f_{2}(x)\right] I_{0}\left[\alpha f_{2}(x)\right]-1\right\},
\end{gathered}
$$

where

$$
f_{2}(x)=x\left(1+\frac{\sin 2 x}{2 x}-2 \frac{\sin ^{2} x}{x^{2}}\right),
$$

and $I_{0}(y)$ is the Bessel function of imaginary argument. Equation (5) suggests that the sign of the correction $\Delta \sigma_{W L}(\Omega)$ is determined by the relation between the times $\tau_{\varphi}$ and $\tau_{s o}$. With strong SOI $\left(\tau_{s o} \ll \tau_{\varphi}\right)$ the following formula should be used:

$$
\Delta \sigma_{W L}(\Omega)=\Delta \sigma(\Omega)-\Delta \sigma(0)=-\frac{1}{2} f_{1}\left(\tau_{\varphi}\right) .
$$

It follows from Eq. (7) that the correction $\Delta \sigma_{W L}(\Omega)$ is negative, which agrees with the results obtained (Figs. 1,2).

For completeness, it is necessary to take into account the possible influence of the ac electric field upon the EEI quantum correction to the conductivity. This influence is enhanced as the temperature goes down. The following expression can be derived from Ref. 1; it describes the corresponding quantum correction for two-dimensional systems at $\hbar \Omega \ll k T$ :

$$
\Delta \sigma_{E E I}(\Omega)=\Delta \sigma(\Omega)-\Delta \sigma(0)=\frac{e^{2}}{2 \pi^{2} \hbar} \frac{7 \zeta(3)}{8 \pi^{2}}\left(\frac{\hbar \Omega}{k T}\right)^{2} .
$$

Note that in this case the presence of the external high-frequency electric field would make the conductivity increase in contrast to its behavior observed on Bi films (Figs. 1,2).

As the above formulas show, the comparison of experimental data and theory requires the knowledge of the $E_{a c}, D$ and $\tau_{\varphi}$ values. The method of estimating $E_{a c}$ proposed in Ref. 7,9 and outlined above has turned out to be ineffective for the samples studied since no pronounced Joule heating was detected in the high-frequency electric field afforded by our experimental facility. For the films with high $R_{\mathrm{n}}$, i.e. about twice as high as $R_{\mathrm{n}}$ of $\mathrm{Bi}-1$ and $\mathrm{Bi}-2(\approx 357 \Omega$ and $\simeq 401 \Omega$, respectively), the influence of the applied ac electric field on the film resistance at $T \simeq 1.5 \mathrm{~K}$ was quite weak (slightly higher than the measurement error). In high-resistance samples the influence of the high-frequency field on the WL effects may be rather small because of short times $\tau_{\varphi}$. The magnitude of the film overheating in the ac electric field is dependent on various parameters and conditions: dissipated power, substrate material, cooling and heat transfer conditions, acoustic coupling of the film and the substrate and so on. The combination of these parameters and conditions for the films studied seems to be that which excludes any appreciable overheating (according to our estimation, not higher than $0.1 \mathrm{~K}$ ) in the applied electric field. Since the resistance measurement using low-frequency current was performed at very low Joule heating, there are reasons to hope that the observed increase in the film resistance in the high-frequency electric field is mainly due to the influence of this field upon the quantum corrections. (Note that, in consequence of the negative resistance coefficient, the Joule heating should cause an opposite effect - a decrease in the resistance of the films.)

The values of time $\tau_{\varphi}$ were determined by analyzing the experimental dependences of the quantum correction on magnetic field. The general expression for the WL-induced variations in conductivity in the two-dimensional systems in a perpendicular magnetic field $H$ can be written down in the form $[1,2]$

$$
\Delta \sigma(H, T)=\frac{e^{2}}{2 \pi^{2} \hbar}\left[\frac{3}{2} f\left(\tau_{\varphi}^{*} / \tau_{H}\right)-\frac{1}{2} f\left(\tau_{\varphi} / \tau_{H}\right)\right],
$$

where $f(x)=\ln x+\Psi(1 / 2+1 / x) ; \Psi$ is the digamma function; $\tau_{H}=\hbar c / 4 D e H$ is the magnetic time. For the strong SOI (typical of Bi films) this expression can be simplified as follows:

$$
\Delta \sigma(H, T)=-\frac{e^{2}}{4 \pi^{2} \hbar} f\left(\frac{\tau_{\varphi}}{\tau_{H}}\right) .
$$

The positive magnetoresistance of the films studied fitted well to this expression for $H \leq 10 \mathrm{kOe}$, and allowed us to estimate the values and temperature dependence of the phase relaxation time $\tau_{\varphi}(T)$.

It should be noted that the processing of the curves $\Delta \sigma(H, T)$ makes it possible to estimate only the values of the product $D \tau_{\varphi}$, so the values of diffusion coeffi- 
cient, $D$, must be known to determine the values of $\tau_{\varphi}$. The values of $D$ are also required to make comparison of the experimental dependences $\Delta \sigma(\Omega)$ with the theory [3]. Therefore, we shall enlarge on our technique of evaluation of $D$ values. To calculate $D$, the commonly accepted expression

$$
D=v_{F}^{2} \tau / 3
$$

was used where $v_{F}$ is the electron velocity at the Fermi level, and $\tau$ is the elastic scattering time. As $\mathrm{Bi}$ is a semimetal with a high-anisotropy electron spectrum, the use of Eq. (11) requires specific justification. Moreover, the electronic characteristics of thin films and bulk samples of Bi differ essentially. Therefore, we have taken account of the fact [13], that the films studied consist of crystallites of small size (somewhat smaller than the film thickness ) and are of axial texture (the axis $C_{3}$ is perpendicular to the film plane). The angular orientation of the crystallites within the film plane is completely random and, hence, the anisotropic properties of individual crystallites do not affect the film conductivity which is quite isotropic. This gives grounds for using the $v_{F}$ value $\left(\simeq 7.5 \cdot 10^{7} \mathrm{~cm} / \mathrm{s}[14]\right)$ averaged in the plane $\left(C_{1} C_{2}\right)$ to calculate $D$. The values of $\tau$ were calculated by the relationship $\sigma=2 n e^{2} \tau / m_{\text {eff }}$ which takes into account the existence of two types of charge carriers in $\mathrm{Bi}$ (electrons and holes) of approximately equal effective mass $m_{\text {eff }} \simeq 5.82 \cdot 10^{-29} \mathrm{~g}[14]$ and concentration $n$. It is known [15] that the concentration of charge carriers in Bi films increases with decreasing film thickness (therefore, the electronic properties of Bi films are much closer to those of typical metals than of bulk $\mathrm{Bi}$ ). The concentration, $n$, was

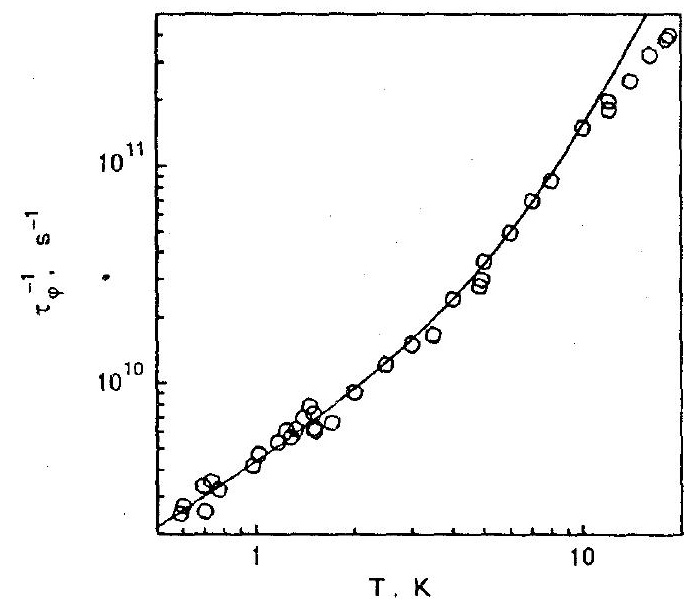

Fig. 3. The temperature dependence of phase relaxation rate $\tau_{\varphi}^{-1}(T)$ for sample $\mathrm{Bi}-1$. The solid line drown through the experimental points corresponds to the expression $\tau_{\varphi}^{-1}(T)=A_{\exp } T+A_{p} T^{3}$. estimated for the films of different thickness, $L$, by using the improved data given in $[16]$. Based on the above facts, we estimated $D$ by $\mathrm{Eq}$. (11) and obtaincd $D=5 \mathrm{~cm}^{2} / \mathrm{s}$ and $\simeq 14 \mathrm{~cm}^{2} / \mathrm{s}$ for samples $\mathrm{Bi}-1$ and $\mathrm{Bi}-2$, respectively.

Figure 3 shows the dependence $\tau_{\varphi}^{-1}(T)$ for samplc $\mathrm{Bi}-1$ resulted from the processing of the experimental curves $\Delta \sigma(H, T)$ with regard to the above value of $D$. The dependence for sample $\mathrm{Bi}-2$ is identical in shape. These dependences (as those in Refs. 12,13) can be well described by the expression $\tau_{p}^{-1}(T)=$ $=A T+A_{p} T^{3}$ where the linear term is the contribution of electron-electron collisions involving small energy transfer $[1,2]$ and the nonlinear term corresponds to the processes of electron-phonon relaxation.

In our study most attention is concentrated on the linear term because it is dominant at low temperatures $T \leq 4.2 \mathrm{~K}$ (Fig. 3) at which one can observe a distinct effect of high-frequency electric field (Figs. 1 and 2). According to Refs. 1,2, the contribution of electron-electron collisions to electron phase relaxation for the two-dimensional case can be given by the relationship

$$
\tau_{\varphi}^{-1}(T)=\frac{\pi k T}{\hbar} \frac{e^{2} R_{\mathrm{n}}}{2 \pi^{2} \hbar} \ln \left(\frac{\pi \hbar}{e^{2} R_{\mathrm{u}}}\right) .
$$

The experimental values of $\tau_{\varphi}$ for the samples studied fit Eq. (12) numerically (to a factor of the order of unity). If we represent Eq. (12) as $\tau_{\varphi}^{-1}=A_{\mathrm{hh}} T$ and the experimental data as $\tau_{\varphi}^{-1}=A_{\exp } T$, the ratio $A_{\text {exp }} / A_{\text {th }}$ equals approximately $=0.66$ and $=1.81$ for samples $\mathrm{Bi}-1$ and $\mathrm{Bi}-2$, respectively. The previous research works $[1,2]$ demonstrate that Eq. (12) which depends only on $R_{\square}$ and the universal constants is well met for metal films (as well as for the $\mathrm{Bi}$ ones $[12,13])$. This indicates that we have made no appreciable error when calculating $D$.

The values of $D$ and $\tau_{\varphi}$ calculated in the above manner were used to compare the experimental values of quantum corrections $\Delta \sigma(\Omega)$ in the presence of high-frequency electric field with the corresponding theoretical expressions $[1,3]$. Our data permit the effect of temperature on $\Delta \sigma(\Omega)$ to be ex"mined more completely and reliably than in the previous works.

The increase in resistance of the Bi films in the presence of high-frequency electric field is consistent with the expected influence of this field on WL effects for strong SOI. Therefore, we decided first of all to use Eq. (7) for comparison with the experimental data. In so doing, according to the experimental results on $\tau_{\varphi}^{-1}(T)$ (Fig. 3) we put $\tau_{\varphi}^{-1}=A T$ and took the experimental and theoretical values $\left(A_{\exp }\right.$ and $A_{\mathrm{th}}$ ) as those of $A$. The dimensionless coefficient $\alpha=$ 


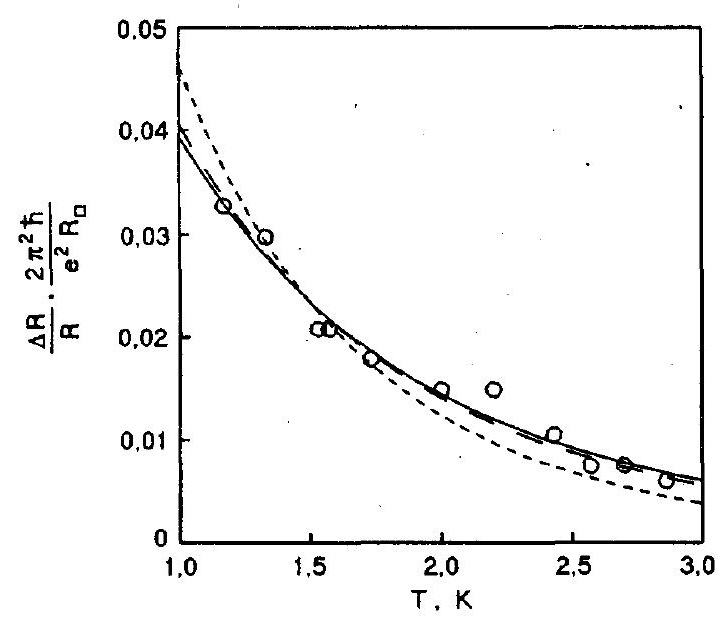

Fig. 4. The temperature dependence of fractional changes in resistance $\Delta R / R$, induced by high-frequency electric field, for sample $\mathrm{Bi}-1$. The difference from Fig. 2, $a$ is that the experimental data for this sample were compared to the sum of corrections $\Delta \sigma_{W L}(\Omega)$ and $и \Delta \sigma_{E E I}(\Omega)$ (Eqs. (7) and (8)). Other details and the versions of calculation used are identical to those in Fig. 2. The values $A_{\exp }, A_{\text {th }}$ and $A_{\text {calc }}$, and those of $\alpha$ and $E_{a c}$ obtained from different versions of calculation are listed in Table 2.

$=4 e^{2} D E_{a c}^{2} / \hbar^{2} \Omega^{3}$ entering into $\mathrm{Eq}$. (7) was a fitting parameter in the search for the best agreement between the experimental and theoretical dependences $\Delta \sigma(\Omega, T)$. In addition, as a third version, this procedure was accomplished with varying both parameters ( $A$ and $\alpha$ ). In that case a certain calculated value of $A$ was determined (we symbolized it as $A_{\text {calc }}$ ). Using the estimated values of $\alpha$ and the above-mentioned values of $D$, we calculated the values of the ac electric field amplitudes $E_{a c}$ in the samples.

The calculated curves of temperature dependences $\Delta \sigma(\Omega, T)$ as well as the values of $\alpha$ and $E_{a c}$ obtained for samples $\mathrm{Bi}-1$ and $\mathrm{Bi}-2$ with different variants of calculation are shown in Fig. 2 and listed in Table 1.

Table 1

\begin{tabular}{c|c|c|c}
\hline Sample & $A, 10^{9} \mathrm{~s}^{-1} \cdot \mathrm{K}$ & $\alpha, 10^{-2}$ & $E_{a c}, \mathrm{~V} / \mathrm{m}$ \\
\hline \multirow{3}{*}{$\mathrm{Bi}-1$} & $A_{\text {exp }}=4.27$ & 5.83 & 50.7 \\
& $A_{\text {th }}=6.49$ & 15.4 & 82.3 \\
& $A_{\text {calc }}=3.20$ & 3.37 & 38.5 \\
\hline \multirow{3}{*}{$\mathrm{Bi}-2$} & $A_{\text {exp }}=1.28$ & 20.4 & 56.7 \\
& $A_{\text {th }}=7.06$ & 3.93 & 24.9 \\
& $A_{\text {calc }}=9.73$ & 8.83 & 37.3 \\
\hline
\end{tabular}

As is seen, the versions of calculation employed result in calculated curves of $\Delta \sigma(\Omega, T)$ that are close together and, in the general case, in close values of field amplitude $E_{a c}$. These values of $E_{a c}$ correspond to the maximum power of microwave radiation for the samples studied (the relation between the observed effects and microwave power will be considered below). It is interesting to note that the version of calculation with varying both parameters independently $(A$ and $\alpha$ ) results in almost identical values $E_{a c} \simeq 38 \mathrm{~V} / \mathrm{m}$ for both samples (Table 1) while the values of quantum corrections $\Delta \sigma(\Omega, T)$ differ approximately by a factor of three.

Table 2

\begin{tabular}{c|c|c|c}
\hline Sample & $A, 10^{9} \mathrm{~s}^{-1} \cdot \mathrm{K}$ & $\alpha, 10^{-2}$ & $E_{a c}, \mathrm{~V} / \mathrm{m}$ \\
\hline $\mathrm{Bi}-1$ & $A_{\exp }=4.27$ & 8.92 & 62.7 \\
& $A_{\text {th }}=6.49$ & 25.3 & 105.6 \\
& $A_{\text {calc }}=3.88$ & 7.31 & 56.7 \\
\hline
\end{tabular}

Although Eq. (7) describes the temperature dependences $\Delta \sigma(\Omega, T)$ quite well, we decided, in contrast to the previous papers [4-9], to take into account the influence of ac electric field on EEI, i.e., to compare the experimental dependences $\Delta \sigma(\Omega, T)$ with a sum of the corrections $\Delta \sigma_{W L}(\Omega)$ and $\Delta \sigma_{E E I}(\Omega)$ (Eqs. (7) and (8)). The result of this calculation for sample Bi-1 is shown in Fig. 4. One can see that the calculated curves of $\Delta \sigma(\Omega, T)$ are arranged almost in the same manner as those in the case where allowance is made only for the term $\Delta \sigma_{W L}(\Omega)$. It should be emphasized that the calculated values of $E_{a c}$ have been varied but not so much. This suggests that within the temperature range under consideration $(1-3 \mathrm{~K})$ the correction $\Delta \sigma_{E E I}(\Omega)$ is small compared to the correction $\Delta \sigma_{W L}(\Omega)$. Thus, with the quantity $\alpha$ as a fitting parameter, our measured temperature dependences $\Delta \sigma(\Omega, T)$ are in good agreement with the known theoretical models $[1,3]$. The values of amplitude $E_{a c}$ calculated using the values of $\alpha$ for the maximum microwave power applied appear to be equal for both samples. This is consistent with what is expected because, according to the electrodynamic calculations [10], at equal amplitude $E_{\text {ext }}$ of incident microwave the amplitude $E_{a c}$ within the thin films depends only on $R_{\mathrm{u}}$. For the samples studied the values of $R_{\mathrm{I}}$ are not too different from one another $(\simeq 357 \Omega$ and $\simeq 401 \Omega$ ), although the values of $D$ differ approximately by a factor of three. It should be noted also that the experimental values of $E_{a c}$ $(40-50 \mathrm{~V} / \mathrm{m})$ are in agreement per se with the estimated values expected.

We obtained additional useful information by measuring the relation between film resistance and incident microwave power (at constant temperatures). 


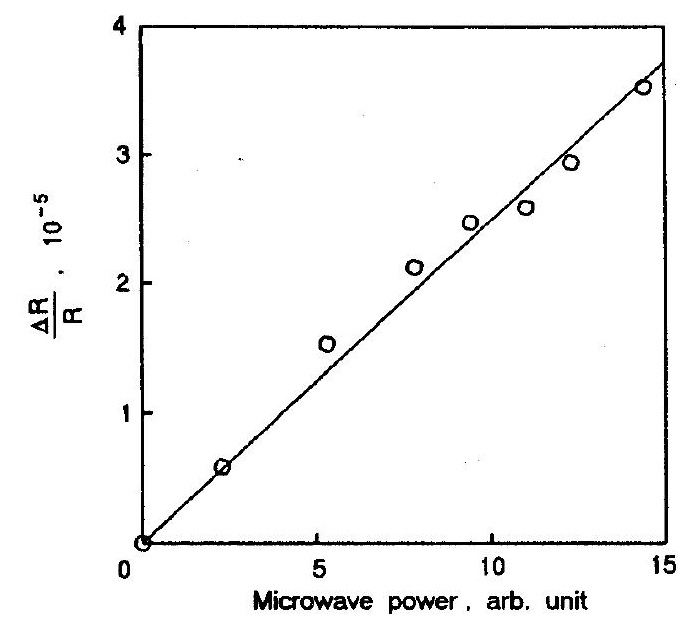

Fig. 5. Changes of film resistance $\Delta R / R$, induced by highfrequency electric field, as a function of incident microwave radiation power at $T=1.2 \mathrm{~K}$.

The relation between resistance increment $\Delta R / R$ and applied power was found to be linear (Fig. 5). This implies that $\Delta \sigma(\Omega, T) \propto \alpha$. This behavior is consistent with the theorctical predictions for low amplitudes of $E_{a c}[6]$

$$
\begin{aligned}
& \Delta \sigma(\Omega, T) \propto \sigma_{0} \alpha\left(\Omega \tau_{\varphi}\right)^{5} \text { at } \alpha\left(\Omega \tau_{\varphi}\right)^{5}<<1, \Omega \tau_{\varphi} \ll<1, \\
& \Delta \sigma(\Omega, T) \propto \sigma_{0} \alpha \Omega \tau_{\varphi} \quad \text { at } \alpha \Omega \tau_{\varphi}<<1, \quad \Omega \tau_{\varphi} \gg>1 .
\end{aligned}
$$

The relationships (13) are valid only with no overheating present. The existence of overheating is supposed to involve (at sufficiently high amplitudes $E_{a c}$ ) an increase in the electron temperature and a decrease in $\tau_{\varphi}$ and hence, a variation in the quantum correction $\Delta \sigma(\Omega, T)$ and thus its departure from linear dependence on microwave power. The absence of such departures for the films studied implies that the effect of overheating is not appreciable. It should be noted that for the above arguments it makes no difference which of relationships (13) is valid for the films studied (the evaluation shows that Eq. (13b) is met) because the very fact of proportionality of $\Delta \sigma(\Omega, T)$ to applied power testifies that there is no appreciable influence of overheating in the films. Therefore, we believe that the comparison between our experimental dependences $\Delta \sigma(\Omega, T)$ and the theoretical models given in Refs. 1,3 is legitimate and the good agreement obtained is quite plausible.

\section{Acknowledgements}

We acknowledge gratefully the experimental assistance by E. Scheer. We thank H. v. Löhneysen and the Sonderforschungsbereich 196 for support, and V. A. Shklovskij for helpful discussion and for providing us with his theoretical results prior to publication.

1. B. L. Al'tshuler, A. G. Aronov, in: Electron-Electron Interactions in Disordered Systems, A. L. Efros and M. Pollak (eds.), Elsevier Sci. Publ., Amsterdam (1985).

2. B. L. Al'tshuler, A. G. Aronov, M. E. Gershenson, and Yu. V. Sharvin, Sov, Sci. Rev. A. Phys. 9, 223 (1987).

3. B. L. Al'tshuler, A. G. Aronov, and D. E. Khmel'nitzkii, Solid State Commun. 39, 619 (1981).

4. M. E. Gershenson and V. N. Gubankov, Pis'ma Zh. Eksp. Teor. Phys. 34, 32 (1981) [JETP Lett. 34, 30 (1981)].

5. S. Wang and P. E. Lindelof, Phys. Rev. Lett. 59, 1156 (1987).

6. S. A. Vitkalov, G. M. Gusev, Z. D. Kvon, G. I. Leviev, and V. I. Fal'ko, Zh. Eksp. Teor. Fiz. 94, 376 (1988) [Sov. Phys. JETP 67, 1080 (1988)].

7. J. Liu and N. Giordano, Phys. Rev. B39, 9894 (1989).

8. J. Liu and N. Giordano, Phys. Rev. B41, 9728 (1990).

9. J. Liu and N. Giordano, Phys. Rev. B43, 1385 (1991).

10. We called our attention to this fact thanks to V. A. Shklovskij who had given proof of this statement by his theoretical results (Not published yet).

11. J. M. Ziman, Principles of the Theory of Solids, Univ. Press, Cambridge (1972).

12. V. Yu. Kashirin and Yu. F. Komnik, Fiz. Nizk. Temp. 18, 1246 (1992) [Sov. J. Low Temp. Phys. 18,872 (1992)].

13. Yu. F. Komnik, V. Yu. Kashirin, B. I. Belevtsev, and E. Yu. Belyaev, Phys. Rev. B50, 15298 (1994).

14. V. S. Edelman, Usp. Fiz. Nauk 123, 257 (1977).

15. Yu. F. Komnik and V. V. Andrievskii, Fiz. Nizk. Temp. 1, 104 (1975) [Sov. J. Low Temp. Phys. 1, 51 (1975)]

16. A. S. Anopchenko, V. Yu. Kashirin, and Yu. F. Komnik, Fiz. Nizk. Temp. 21, 451 (1995) [Low Temp. Phys. 21, 353 (1995)]. 


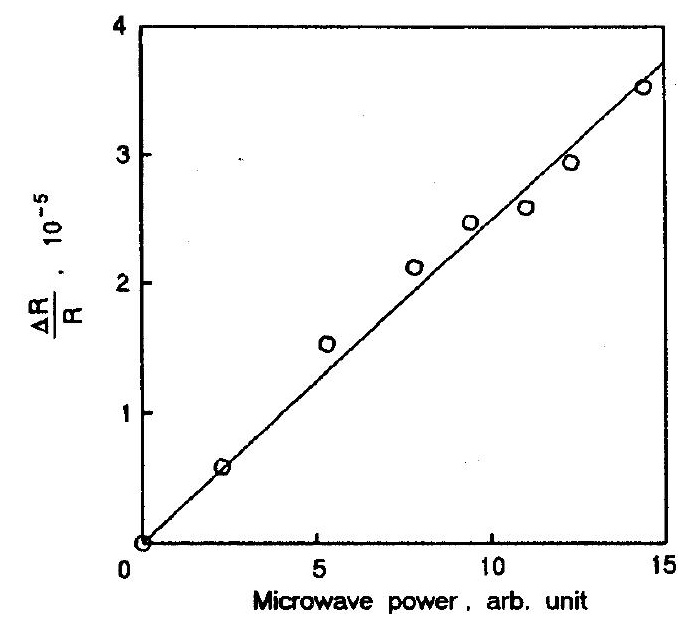

Fig. 5. Changes of film resistance $\Delta R / R$, induced by highfrequency electric field, as a function of incident microwave radiation power at $T=1.2 \mathrm{~K}$.

The relation between resistance increment $\Delta R / R$ and applied power was found to be linear (Fig. 5). This implies that $\Delta \sigma(\Omega, T) \propto \alpha$. This behavior is consistent with the theorctical predictions for low amplitudes of $E_{a c}[6]$

$$
\begin{aligned}
& \Delta \sigma(\Omega, T) \propto \sigma_{0} \alpha\left(\Omega \tau_{\varphi}\right)^{5} \text { at } \alpha\left(\Omega \tau_{\varphi}\right)^{5}<<1, \Omega \tau_{\varphi} \ll<1, \\
& \Delta \sigma(\Omega, T) \propto \sigma_{0} \alpha \Omega \tau_{\varphi} \quad \text { at } \alpha \Omega \tau_{\varphi}<<1, \quad \Omega \tau_{\varphi} \gg>1 .
\end{aligned}
$$

The relationships (13) are valid only with no overheating present. The existence of overheating is supposed to involve (at sufficiently high amplitudes $E_{a c}$ ) an increase in the electron temperature and a decrease in $\tau_{\varphi}$ and hence, a variation in the quantum correction $\Delta \sigma(\Omega, T)$ and thus its departure from linear dependence on microwave power. The absence of such departures for the films studied implies that the effect of overheating is not appreciable. It should be noted that for the above arguments it makes no difference which of relationships (13) is valid for the films studied (the evaluation shows that Eq. (13b) is met) because the very fact of proportionality of $\Delta \sigma(\Omega, T)$ to applied power testifies that there is no appreciable influence of overheating in the films. Therefore, we believe that the comparison between our experimental dependences $\Delta \sigma(\Omega, T)$ and the theoretical models given in Refs. 1,3 is legitimate and the good agreement obtained is quite plausible.

\section{Acknowledgements}

We acknowledge gratefully the experimental assistance by E. Scheer. We thank H. v. Löhneysen and the Sonderforschungsbereich 196 for support, and V. A. Shklovskij for helpful discussion and for providing us with his theoretical results prior to publication.

1. B. L. Al'tshuler, A. G. Aronov, in: Electron-Electron Interactions in Disordered Systems, A. L. Efros and M. Pollak (eds.), Elsevier Sci. Publ., Amsterdam (1985).

2. B. L. Al'tshuler, A. G. Aronov, M. E. Gershenson, and Yu. V. Sharvin, Sov, Sci. Rev. A. Phys. 9, 223 (1987).

3. B. L. Al'tshuler, A. G. Aronov, and D. E. Khmel'nitzkii, Solid State Commun. 39, 619 (1981).

4. M. E. Gershenson and V. N. Gubankov, Pis'ma Zh. Eksp. Teor. Phys. 34, 32 (1981) [JETP Lett. 34, 30 (1981)].

5. S. Wang and P. E. Lindelof, Phys. Rev. Lett. 59, 1156 (1987).

6. S. A. Vitkalov, G. M. Gusev, Z. D. Kvon, G. I. Leviev, and V. I. Fal'ko, Zh. Eksp. Teor. Fiz. 94, 376 (1988) [Sov. Phys. JETP 67, 1080 (1988)].

7. J. Liu and N. Giordano, Phys. Rev. B39, 9894 (1989).

8. J. Liu and N. Giordano, Phys. Rev. B41, 9728 (1990).

9. J. Liu and N. Giordano, Phys. Rev. B43, 1385 (1991).

10. We called our attention to this fact thanks to V. A. Shklovskij who had given proof of this statement by his theoretical results (Not published yet).

11. J. M. Ziman, Principles of the Theory of Solids, Univ. Press, Cambridge (1972).

12. V. Yu. Kashirin and Yu. F. Komnik, Fiz. Nizk. Temp. 18, 1246 (1992) [Sov. J. Low Temp. Phys. 18,872 (1992)].

13. Yu. F. Komnik, V. Yu. Kashirin, B. I. Belevtsev, and E. Yu. Belyaev, Phys. Rev. B50, 15298 (1994).

14. V. S. Edelman, Usp. Fiz. Nauk 123, 257 (1977).

15. Yu. F. Komnik and V. V. Andrievskii, Fiz. Nizk. Temp. 1, 104 (1975) [Sov. J. Low Temp. Phys. 1, 51 (1975)]

16. A. S. Anopchenko, V. Yu. Kashirin, and Yu. F. Komnik, Fiz. Nizk. Temp. 21, 451 (1995) [Low Temp. Phys. 21, 353 (1995)]. 